\title{
THE INFLUENCE OF DIFFERENT STRATEGIES ON THE PRODUCTION OF OIL FIELDS STIMULATED BY WATERFLOODING
}

\begin{abstract}
R. F. Martinia,
J. S. de A. Cavalcante Filho ${ }^{\text {, }}$, and D. J. Schiozer ,

${ }^{\mathrm{a}, \mathrm{b}}$ PETROBRAS

Av. República do Chile, 65

20031-912, Rio de Janeiro, RJ, Brasil

'arogerio.martini@petrobras.com.br

Av. Euzébio Rocha, 1000

59070-660, Natal, RN, Brasil

bsergio.cavalcante@petrobras.com.br

${ }^{c} \mathrm{UNICAMP} / \mathrm{FEM} / \mathrm{DEP}$

Caixa Postal 6122

13083-970, Campinas, SP, Brasil

\section{ABSTRACT}

Quality map is an important tool which can assist in the definition and refinement of production strategies, since it indicates the production potential of each place of the reservoir, combining several parameters that influence oil recovery efficiency. This work has used numerical simulation to analyse the performance of different production strategies used in waterflood projects for an offshore reservoir; these strategies included peripheral injection/central production, a 5-spot injection/production scheme and a strategy defined with the assistance of a quality map; after the initial process of strategy definition, all schemes were optimised. The refinement procedure was assisted by an expert system, which proposes well modifications based on the analysis of economic and technical parameters and stores the analysed parameters in a databank. The results show that the use of the quality map improved to a great extent the processes of strategy definition and refinement, yielding better results and requiring fewer simulation runs. The application of quality maps to petroleum reservoirs is still quite recent, with very few works published; however, they present great potential for the E\&P industry, as presented here.
\end{abstract}

denis@dep.fem.unicamp.br
Keywords: Production strategies, Quality maps, Waterflood, Horizontal wells.

\section{NOMENCLATURE}

FR Recovery Factor, \%

MQ Quality Map

NPV Net Present Value, MMUS\$

\section{INTRODUCTION}

The efficient management of reservoirs encompasses the setting of the best production strategy, taking into account physical, operating and economic restrictions. Due to the large number of variables involved, however, the definition of production strategies is often a long and subjective process where reservoir engineers are usually faced with a set of possible options, instead of a unique solution. Among these variables are the reservoir characteristics, the number and type of wells coupled with their operating conditions plus the geological uncertainties.

The constant search for cost reduction, productivity maximization and extension of the production lifetime of reservoirs has been aided by the technological development of the last few years. Advances in perforation and completion techniques have made possible the use of horizontal wells, which present important advantages over vertical ones (Mascarenhas and Durlofsky, 2000). Among these advantages one could quote their higher productivity and capacity of increasing reserves, resultant of their greater length when compared to vertical wells. The larger area of contact with the producer layer also yields a more complex interaction between the reservoir and the well, thus using horizontal wells might increase either the potential of success or failure of the strategy; for example, in reservoirs containing aquifers, an horizontal well crossing a region of high vertical permeability might present early water breakthrough in some sections (Raghuraman et al., 2003). Therefore, thorough studies of the several parameters affecting the behaviour of such wells are essential to achieve the goals set by the management team.

The proper identification of the more suitable regions for production is no simple task, since there are numerous parameters governing fluid flow through reservoirs. It is not easy to predict the reservoir behaviour during production even when all the parameters are available separately, especially when dealing with heterogeneous reservoirs, due to the complex, non-linear interaction between these parameters (Cruz et al., 2004). For instance, given a reservoir, one must consider the presence (or absence) of gas cap and aquifer, besides their distance to the wells; large horizontal permeability values in the production layers are favourable to oil extraction, whereas smaller vertical permeability values between the production layers and an aquifer may be desirable to reduce water production (Cruz, 2000). Other important variables are the saturation (of oil, gas and/or water), the porosity and the relative permeability. 


\section{PRODUCTION STRATEGY DEFINITION AND REFINEMENT}

Projects of reservoir recovery can be divided into two main stages (or phases) regarding production strategy: the first phase is marked by the existence of great uncertainty in the values of the reservoir properties, when the strategy is defined and the numerical simulation models are simpler; simulation runs are shorter, however, there is a broad range of possible schemes; Mezzomo and Schiozer (2003) proposed a methodology for strategy definition which divided this phase in six steps, as shown in Fig. 1; the purpose of such a methodology is to allow the definition of feasible production strategies for the development of new fields; the first step is characterised by the largest number of possible strategies and at each stage the less suitable ones are discarded, which guarantees that only the best strategies remain when step number 6 is concluded. The next (seventh) step (Schiozer and Mezzomo, 2003) is part of the second phase, which is marked by lower uncertainty in the reservoir properties and more complex simulation models.

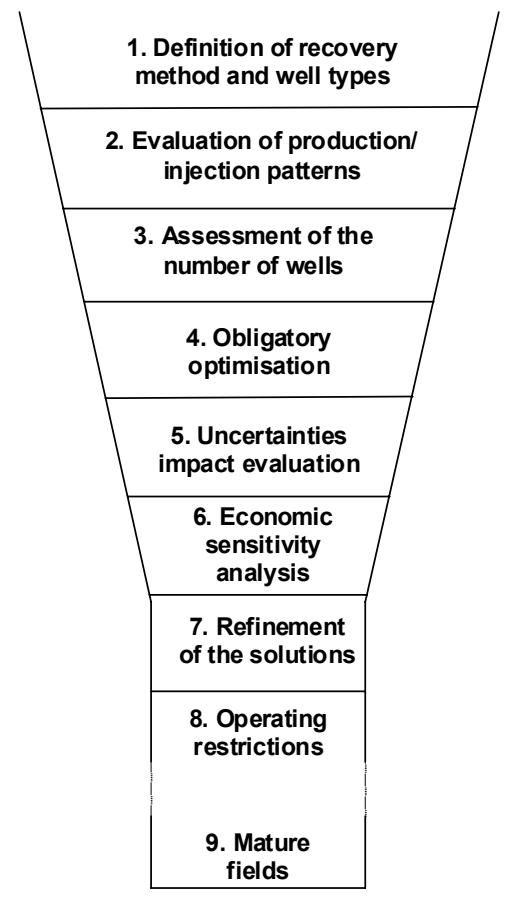

Figure 1. Methodology for strategy definition and refinement

The aim of the refinement process in steps 7 to 9 is to attain the best production configuration, considering the company's goals, which are represented in the refinement process by the minimization (or maximization) of an objective function, e.g., the net present value, oil, water and gas cumulative production, or even a combination of these parameters; step $n^{\circ} 7$ concerns the refinement of the strategies defined in steps 1-6 and it is characterised by a reasonably wide range of alternatives for well modifications, whereas in step 8 one must consider restrictions such as platform maximum capacity for oil storage or water treatment, conditions for raising the oil to the platform and so on. Step 9 has the characteristic of involving a reservoir which is better known due to the data gathered during the several years of production, however the production strategy is already established and there are fewer options for modifications. The work presented here refers to step $n^{\circ} 7$ in the sense that the reservoir characteristics are known and no uncertainty analysis was performed.

\section{QUALITY MAPS}

Conventional 2D and 3D maps can show one property at a time, for instance oil saturation; therefore, during the stages of production strategy definition and refinement, engineers must analyse several maps in order to predict the reservoir behaviour and probable performance during production. Quality maps represent an important tool as they can combine several parameters, e.g. oil saturation, cell porosity and relative permeability, in one graphical representation. This 2D representation works as a measure of the potential for production of each area in the reservoir and, since it aggregates static and dynamic parameters such as cell porosity and oil saturation, quality maps change during the production process as a reflection of the changes in those properties (Nakajima, 2003). There are different ways of generating these maps (Nakajima and Schiozer, 2003) and more research work on this subject is ongoing, however, the best consolidated method to the present is the generation of quality maps by numerical simulation. Numerical simulation can properly evaluate the productivity of a well, since it can take into account the several variables which it depends upon and the highly non-linear dependence between them. Maps can be generated either by using simulation of vertical or horizontal wells, individually or in groups (Nakajima, 2003), and the well productivity can be assessed in terms of a technical parameter such as the cumulative oil production, $\mathrm{Np}$, or an economic parameter, e.g., the net present value, NPV. The results thus obtained are normalized, yielding the "quality factor" (or "index"), which is a parameter varying from 0 (zero) to 1 (one); the closer to 1 , the better the production potential. The whole reservoir must be evaluated, meaning that several simulation runs are usually necessary to generate a quality map. 


\section{PRODUCTION STRATEGY DEFINITION}

Three different well patterns, all using horizontal wells both for injection and production, were studied: a 5-spot pattern, a peripheral injection/central production (hereafter cited as "peripheral"), and a well configuration based on a quality map (referred to as MQ).

An offshore reservoir was simulated using a commercial black-oil simulator; the model grid was composed of $80 \times 45 \times 22$ blocks and the total simulation time was 9862 days (27 years) in all runs. The definition of strategies for the 5-spot and the peripheral patterns started with a single well, allocated in a region of the reservoir with high oil saturation; more wells were added gradually to the subsequent runs, in intervals of 90 days, according to the pattern previously defined and the observed reservoir oil saturation maps (producers were allocated in areas of high oil saturation and the injectors position was chosen according to the reservoir pressure behaviour). Figure 2 shows the economic behaviour of the field (measured by the net present value) during the simulation and as wells were added; a total of twelve simulations were run. The initial configuration (1A) and the best one (10A) were plotted with markers to allow better visualisation of the process, where many attempts resulted in worse performance (lower NPV); run 10A involves the use of 8 producer wells and 4 injectors and was taken to the next stage, of strategy refinement.

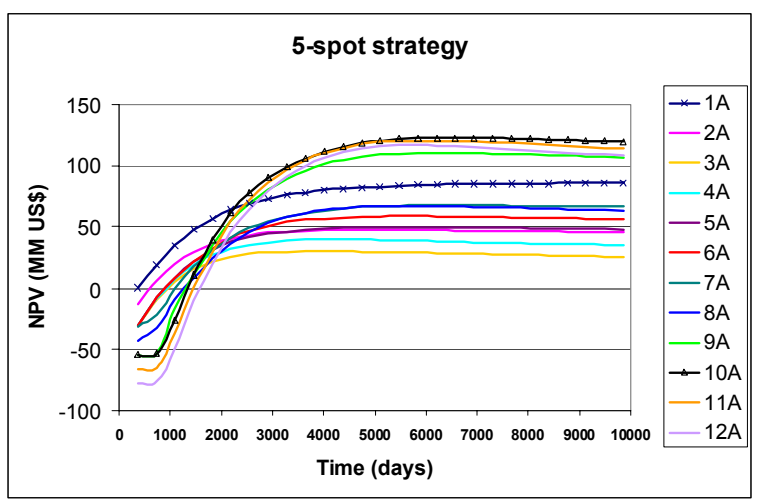

Figure 2. Behaviour of Net Present Value with time and runs (5-spot)

Similarly to the 5-spot case, the definition of the peripheral strategy started with a single well and others were added according to the peripheral pattern and the oil saturation maps; Fig. 3 shows the evolution of NPV with time for each run; simulation run $1 \mathrm{~A}$ was the starting point, and again run $10 \mathrm{~A}$ presented the best performance, which was then taken as the base case to the next stage (refinement); in this case, there are 7 producers and 3 injectors.

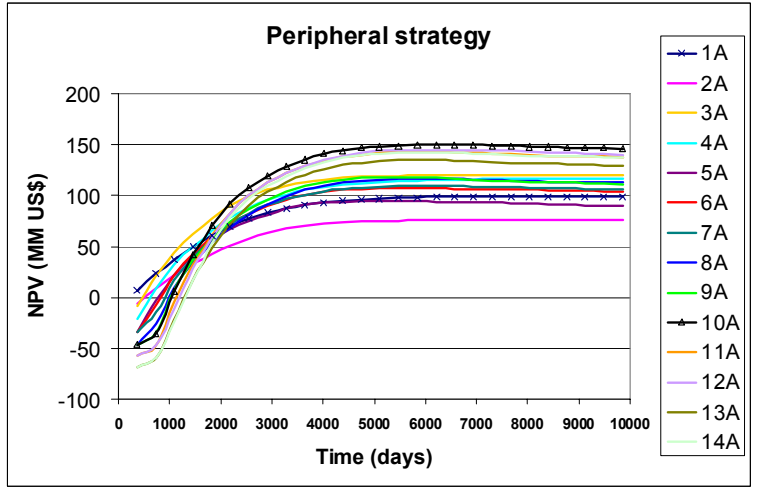

Figure 3. Behaviour of Net Present Value with time and runs (peripheral)

With the MQ strategy, a quality map was initially generated and then used to allocate the initial number of wells, which formed the base case of the next stage; in this case, it was not necessary to add wells one by one as the quality map already displayed the best regions; 8 producers and 4 injectors were used, to start with a total number of wells equivalent to the ones from the previous cases. The map, which can be seen in Fig. 4, was generated by a single simulation run with 51 wells spread through the reservoir (run 1A). The parameter used to indicate the block quality factor was the net present value (NPV). The red colour indicates the areas with best production potential; the green area represents regions with intermediate potential and the blue colour signals areas with poor production potential. The producer wells were then allocated in the red area, with the injectors positioned in the green area, near the border between green and blue. The axes of the quality map are the areal (XY) grid block coordinates.

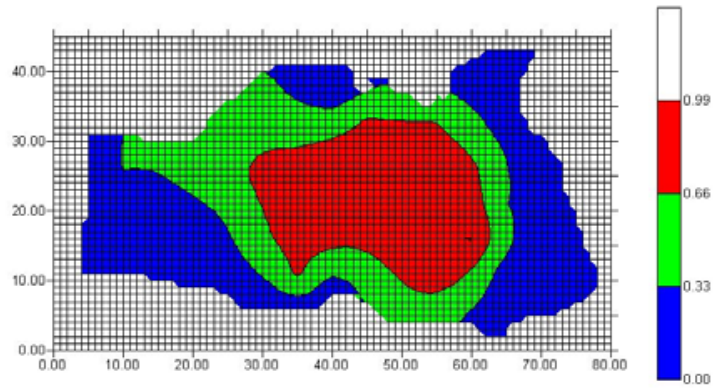

Figure 4. Quality map generated by 1 simulation run with 51 wells

Table 1 shows a summary of the cases which presented better performance in the 5-spot and peripheral strategies, which were taken to the next stage as base cases; the initial strategy defined solely with the aid of the quality map is also presented; it is possible to observe that the financial performance of this configuration (MQ), despite being worse than the other two cases, shows already a positive value for 
NPV and a cumulative production of oil which is not too different from those obtained after several simulation runs in the other case studies.

Table 1. Summary of cases to be used as a basis for the refinement process

\begin{tabular}{|c|c|c|c|c|}
\hline Strategy & $\begin{array}{c}\text { Number } \\
\text { of wells }\end{array}$ & $\begin{array}{c}\text { Number } \\
\text { of runs }\end{array}$ & $\begin{array}{c}\text { NPV } \\
\text { (MM US\$) }\end{array}$ & $\begin{array}{c}\mathrm{Np} \\
\left(10^{6} \mathrm{~m}^{3}\right)\end{array}$ \\
\hline 5-spot & 12 & 12 & 120 & 39.61 \\
\hline Peripheral & 10 & 14 & 146 & 38.57 \\
\hline $\begin{array}{c}\text { Quality } \\
\text { Map (MQ) }\end{array}$ & 12 & 1 & 115 & 31.32 \\
\hline
\end{tabular}

\section{PRODUCTION STRATEGY REFINEMENT}

Refinement works often use algorithms to achieve their goals, where the user's job usually is to evaluate the final answer, checking for consistency. Recently, some authors have made use of refinement procedures such as genetic algorithms (GA): Bittencourt and Horne (1997) and Montes et al. (2001) resorted to GA to help in the search for the best location for wells, whereas Guyaguler and Horne (2000) presented a refinement procedure based on a hybrid genetic algorithm, which reduced the number of simulations when compared to simple GA.

The refinement process presented in this work follows a different approach, where an expert system is used to assess the field and wells performance, then suggest modifications to the user, who can carry them out or decide for other changes; it is, therefore, an interactive process which aims at taking advantage of both a robust problem analysis and the user's experience and knowledge of reservoir engineering. The analysis performed by the system is based on the parameters below; the data from parameter $\mathrm{N}^{\circ} 6$ was not used for strategies 5-spot and peripheral, as these were defined and optimised without any assistance from a quality map:

1. Net present value of each producer well (NPV),

2. Cumulative oil production of each producer $(\mathrm{Np})$,

3. Cumulative water production of each producer (Wp),

4. Cumulative gas production of each producer (Gp),

5. Average oil production rate (Qom),

6. Data from the quality map (Mp).

This methodology was applied to the base cases from the 5-spot, peripheral and MQ configurations; modifications were made to the wells pointed out by the expert system; next, the simulation models were run again and a new analysis was made; this procedure was repeated until the improvement in the field performance became negligible in comparison to the effort spent to attain it, that is, many modifications followed by simulation runs would result in little increase of NPV. Another parameter considered was the recovery factor (FR): a lower limit of $35 \%$ to $40 \%$ was set for these simulations and this kind of reservoir.

Figure 5 shows the strategies tested for the 5spot pattern; "Strategy A" refers to the simulation runs used to define the production strategy, which were described in the previous section. The best performing run (10A) was used as the base case for the refinement (strategy paths $\mathrm{B}, \mathrm{C}$ and $\mathrm{D}$ ). The highest NPV achieved occurred in run $14 \mathrm{~B}$ and 10 simulations were run in the refinement stage of this case study.

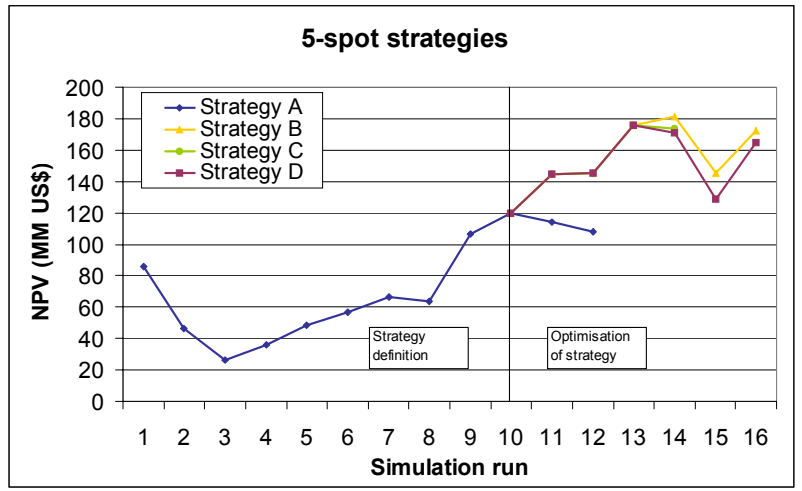

Figure 5. Field performance in terms of Net Present Value (5-spot configuration)

The peripheral strategy results are presented in Fig. 6; again, "Strategy A" refers to the definition phase, whereas four different strategies were tried in the refinement stage, accounting for 11 simulation runs. Simulation 10A was used as the base case for the refinement, and the best configuration was achieved in run $17 \mathrm{E}$.

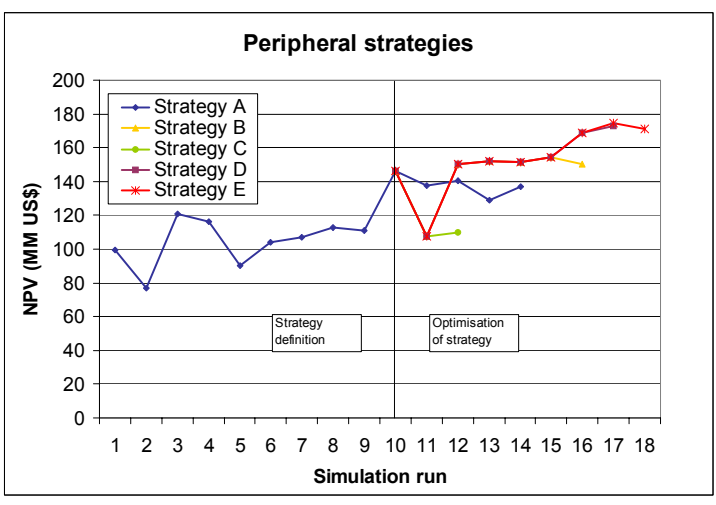

Figure 6. Field performance in terms of Net Present Value (peripheral configuration)

Figure 7 presents the paths followed during the refinement of the strategy assisted by the quality map; a single run was necessary to define the strategy, and 15 simulations to optimise it; the best result was obtained in simulation run $10 \mathrm{E}$ (which was very close to runs $8 \mathrm{E}$ and $9 \mathrm{E}$ ). 


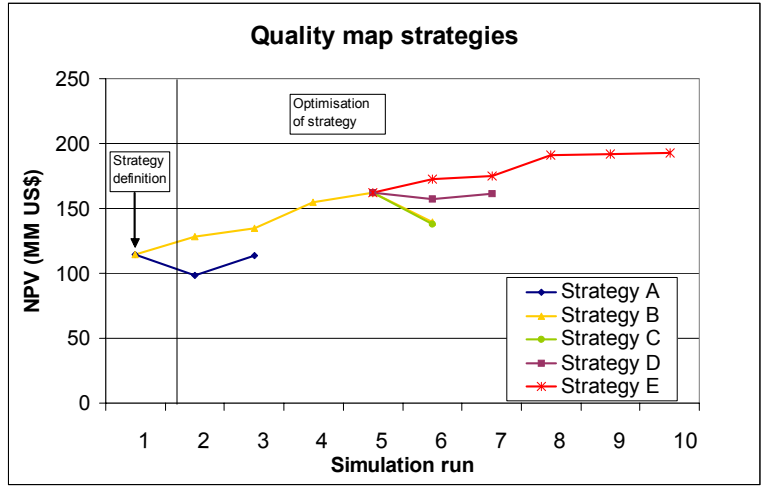

Figure 7. Field performance in terms of Net Present Value (MQ configuration)

The comparison between the three cases can be seen at Fig. 8, which shows the NPV evolution during the simulation time for the three base cases (beginning of the refinement) and for the best production strategies obtained, using the 5-spot pattern, the peripheral injection/central production configuration and the strategy optimised with the assistance of the quality map. There was a considerable difference between the starting point in the peripheral configuration and the other two base cases (5-spot and MQ), however this difference was reduced significantly during the refinement: the 5spot and peripheral patterns present very similar NPV profiles, whereas the MQ strategy performance is slightly better (higher NPV).

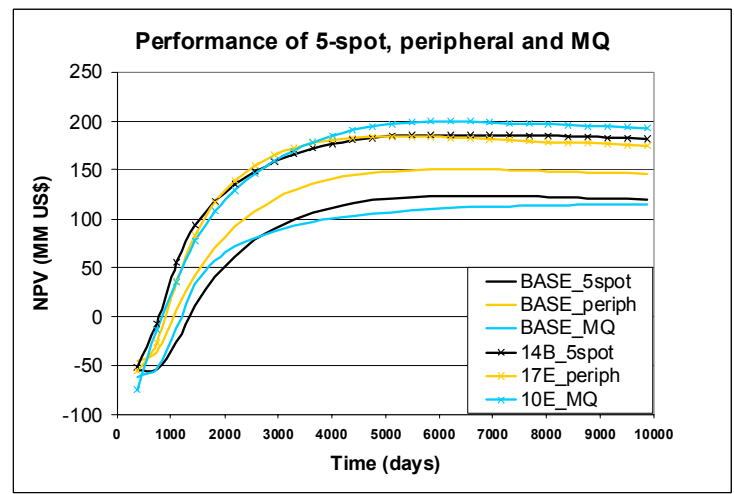

Figure 8. Field performance - base cases and best strategies of all configurations (5-spot, peripheral and MQ)

Considering only the refinement stage, it took the MQ strategy more runs to achieve the final result; however, when the steps needed to define the base cases are taken into account, one can see that the strategy assisted by the quality map was the fastest one, as can be seen in Tab. 2, where the total number of runs is the sum of runs needed in the first stage (definition) and the second stage (refinement). For the 5-spot and peripheral patterns, simulation runs other than the ones shown in Tab. 2 achieved higher recovery factors; however, the field NPV in those cases was lower due to the concomitant higher production of water. In the MQ case, the data shown in Tab. 2 represent both the highest NPV and Np obtained in the simulations.

Table 2. Final refinement results

\begin{tabular}{|c|c|c|c|c|}
\hline $\begin{array}{c}\text { Optimised } \\
\text { Strategy }\end{array}$ & $\begin{array}{c}\text { Total } \\
\text { number } \\
\text { of runs }\end{array}$ & $\begin{array}{c}\text { NPV } \\
\text { (MM US\$) }\end{array}$ & $\begin{array}{c}\mathrm{Np} \\
\left(10^{6} \mathrm{~m}^{3}\right)\end{array}$ & $\begin{array}{c}\text { Recovery } \\
\text { factor (\%) }\end{array}$ \\
\hline 5-spot & 22 & 181 & 41.20 & 35.72 \\
\hline Peripheral & 25 & 175 & 44.64 & 38.71 \\
\hline $\begin{array}{c}\text { Quality } \\
\text { Map (MQ) }\end{array}$ & 16 & 193 & 49.44 & 42.86 \\
\hline
\end{tabular}

\section{CONCLUSIONS}

Three different production patterns were compared: 5-spot, peripheral injection/central production and a quality map-assisted configuration. The results show that, after refinement, different production patterns can achieve similar results, even when there is a considerable difference between the initial strategies.

It has been shown that the quality map can be a valuable tool in the definition and refinement of production strategies, by providing visual information of the reservoir regions with best production potential, therefore reducing the time needed to define feasible strategies and helping the refinement procedure to achieve better results.

The methodology followed in this work makes use of an expert system to assist with the refinement of production strategies, allowing users to interact closely with the problem and decide which path (or paths) to follow; the database generated by the system makes it possible to compare the performance of wells relative to each other and the field performance of all the simulation runs. The well modifications are suggested not only for the worst ones, but also for wells presenting good performance, in order to further improve their results.

\section{ACKNOWLEDGEMENTS}

The authors are grateful to Conselho Nacional de Desenvolvimento Científico e Tecnológico (CNPq), Agência Nacional do Petróleo (ANP), Universidade Estadual de Campinas (UNICAMP) and PETROBRAS for their financial support.

\section{REFERENCES}

Bittencourt, A.C. and Horne, R.N., 1997, Reservoir Development and Design Optimization, in: SPE Annual Technical Conference and Exhibition, San Antonio, Texas, USA, October 5-8, SPE 38895.

Cruz, P.S., 2000, Reservoir Management Decision-Making In The Presence Of Geological Uncertainty, Doctoral Thesis, University of Stanford, Palo Alto, CA. 
Cruz, P.S., Horne, R.N. and Deutsch, C.V., 2004, The Quality Map: A Tool for Reservoir Uncertainty Quantification and Decision Making, SPE Reservoir Evaluation and Engineering, Vol. 7, No. 1, pp. 6-14, SPE 87642.

Guyaguler, B. and Horne, R., 2000, Optimization of Well Placement, Journal of Energy Resources Technology - Transactions of the ASME, Vol. 122, No. 2, pp. 64-70.

Mascarenhas, O. and Durlofsky, L.J., 2000, Coarse scale simulation of horizontal wells in heterogeneous reservoirs, Journal of Petroleum Science and Engineering, Vol. 25, No. 3-4, pp. 135147.

Mezzomo, C.C. and Schiozer, D.J., 2003, Methodology for Water Injection Strategies Planning Optimization Using Reservoir Simulation, Journal of Canadian Petroleum Technology, Vol. 42, No. 7, pp. 9-11.

Montes, G., Bartolome, P. and Udias, A.L., 2001, The Use of Genetic Algorithms in Well Placement Optimization, in: SPE Latin American and Caribbean Petroleum Engineering Conference, Buenos Aires, Argentina, March 25-28, SPE 69439.

Nakajima, L., 2003, Horizontal Wells Performance Optimization on Petroleum Fields Development, Masters Thesis, State University of Campinas, Campinas, SP, Brazil.

Nakajima, L. and Schiozer, D.J., 2003, Horizontal Well Placement Optimization Using Quality Map Definition, in: Petroleum Society's Canadian International Petroleum Conference, Calgary, Alberta, Canada, CIPC 2003-053, June 1012.

Raghuraman, B., Couet, B. and Savundararaj, P., 2003, Valuation of Technology and Information for Reservoir Risk Management, SPE Reservoir Evaluation and Engineering, Vol. 6, No. 5, pp. 307315, SPE 86568.

Schiozer, D.J. and Mezzomo, C.C., 2003, Methodology for Field Development Optimization with Water Injection, in: SPE Hydrocarbon Economics and Evaluation Symposium, Dallas, Texas, USA, SPE 82021.

Received: February 05, 2006

Revised: March 05, 2006

Accepted: April 05, 2006 